\title{
Birds Do It, Bees Do It, We Do It: Contributions of Theoretical Modelling to Understanding the Shape of Ageing across the Tree of Life
}

\author{
Annette Baudisch \\ Max Planck Research Group 'Modelling the Evolution of Ageing', Max Planck Institute for Demographic Research, \\ Rostock, Germany
}

\section{Key Words}

Ageing $\cdot$ Trade-offs $\cdot$ Modelling $\cdot$ Mortality $\cdot$ Theory $\cdot$ Comparative approach

\begin{abstract}
Organisms of different species age differently. Current theory explains why life should get worse, i.e. why patterns of increasing risk of death should evolve. However, for some species the risk of death remains constant or even falls with advancing age. Evolutionary theory to explain the observed diversity of shapes of ageing is lacking. Theoretical models can provide insights into this diversity. Comparing assumptions of models that find increasing mortality patterns with models that find a variety of patterns, including constant and falling mortality over age, I identify conditions that licence constant or negative shapes of ageing. The results suggest that patterns of improvement and maintenance over age emerge when models potentially allow organisms to (1) escape the 'damage ratchet', (2) achieve maintenance and repair in parallel, (3) face increasing future reproductive potential and (4) incorporate flexible trade-offs. With these insights, theoretical models contribute to hypotheses about which species may follow life history strategies of negligible or negative ageing.

Copyright $\odot 2012$ S. Karger AG, Basel
\end{abstract}

\section{KARGER}

E-Mail karger@karger.ch www.karger.com/ger (c) 2012 S. Karger AG, Basel Karger
0304-324X/12/0586-0481\$38.00/0 Open access
This is an Open Access article licensed under the terms of This is an Open Access article licensed under the terms of Derivs 3.0 License (www.karger.com/OA-license), applicable to the online version of the article only. Distribution for non-commercial purposes only.

\section{Introduction}

Why do birds age like birds and bees age like bees? What do species have in common, what is different and why did ageing evolve that way for them? Which species can be taken as a model for human ageing?

Deeply rooted in human experience, the term 'ageing' is loaded with associations of physiological decay and loss of functioning and vitality. In 1952, Sir Peter Medawar noted that the English language curiously lacks a term that captures 'the mere increase of years ... with no other innuendo'. He suggested using 'ageing' for mere ageing and to reserve the term 'senescence' for 'ageing accompanied by the decline of bodily faculties and sensibilities and energies which ageing colloquially entails' [1].

In Medawar's terms, all species age, i.e. they change over age, but do all species senesce? Do all species, just like humans, inevitably proceed along a path of decay and decrepitude? To study ageing within a comparative framework, it needs to be defined in a way that is applicable to species that differ vastly in their physiology and genetic make-up. Common to all species is the currency of birth and death rates. They determine population dynamics and structure, i.e. demography, and thereby drive evolution. Evolution, in turn, selects those genotypes as- 
sociated with patterns of birth and death that achieve maximum population growth and thus fitness. Marginal fitness benefits due to heritable changes in a trait hinge on its effect on mortality and fertility. Demography drives evolution, and evolution shapes demography.

Specifically here then, 'ageing' is defined as a change in mortality and fertility over adult ages. This definition embraces both beneficial and detrimental changes with age $[1,2]$. If organisms experience increasing death rates with age, they are said to exhibit 'senescence' [3]. Organisms that show constant or falling mortality patterns have been classified as showing 'negligible senescence' $[3,4]$ or 'negative senescence' $[2,5]$. Constant or decreasing death rates can broadly be referred to as non-senescence. An analogous definition holds for reproductive patterns.

Negligible or negative senescence should not be misunderstood as meaning to escape death. A non-senescent organism would still die, just not from a progressive loss of functioning and vitality. Accidents, diseases and famine pose a ubiquitous hazard that can strike at any age. In a hazardous environment, both senescent as well as nonsenescent organisms can live only for a short time. Thus, what determines life span (pace) versus what determines the qualitative pattern of ageing (shape) need to be carefully distinguished [6] (box 1).

\section{Box 1: Pace versus Shape of Ageing}

Senescence and life span capture two distinct aspects of ageing. Often, long-lived species are suspected to be those that show the least senescence, but this need not be the case. Though closely connected, ageing is not only about the length of life. Independent of whether a creature measures its life span in days or centuries, death rates may remain the same, go up or fall over the course of life [6]. Also, mortality can change by only a few percentage points or it might change several-fold as organisms get older. For example, while mortality in Swedish females in 2007 increased on average 35fold over adult life, for the much shorter-lived, freeranging African buffalo, mortality increased only 12fold. Swedes have a slower pace but a steeper shape of senescence compared to buffalos [6]. Studying the evolution of ageing thus implies two questions: what determines the length of life, i.e. its pace, and what determines the direction and magnitude of change in mortality (and fertility) over age, i.e. the shape of ageing (fig. 1).

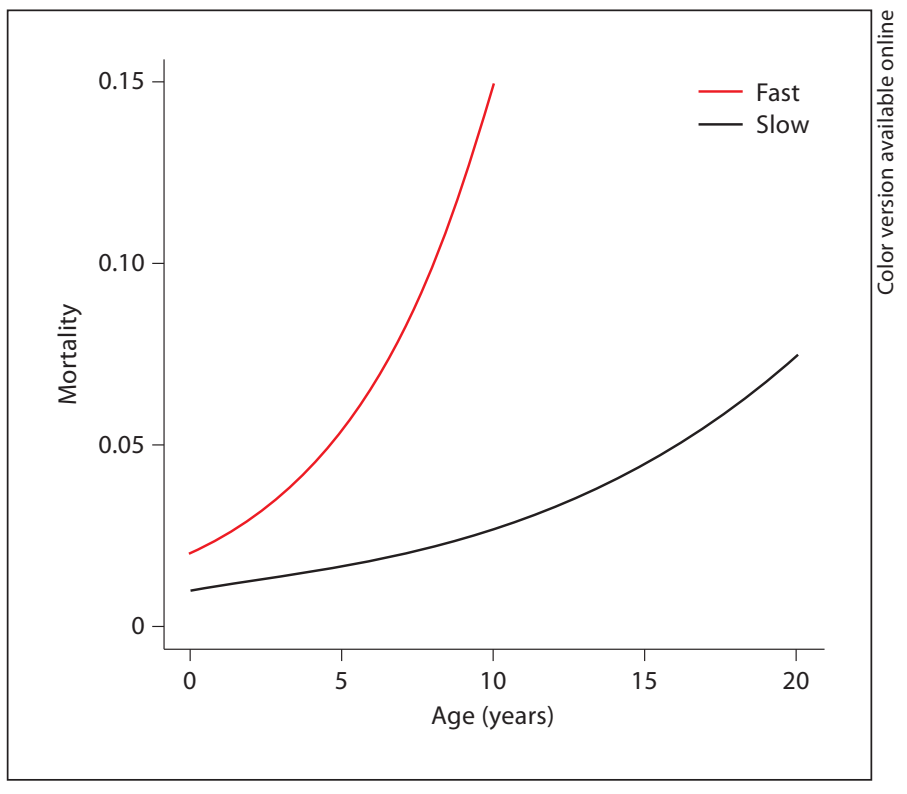

Fig. 1. Example of mortality patterns for two species that differ in pace but share the same shape of ageing. The 'fast' species lives at twice the speed of the 'slow' species, with a respective life expectancy of 10 versus 20 years. For both species, over the expected length of life, mortality increases 7.5-fold; in the former from a level of 0.02 to 0.15 , and in the latter from 0.01 to 0.075 . Examples are calculated assuming Gompertz mortality, given by $a \exp (b x)$, with parameters $a=0.1$ and $b=0.1$ for the slow species and $a=$ 0.2 and $b=0.2$ for the fast species.

Given a certain environmental hazard of death, whether or not and in what way a species should exhibit senescence depends on physiological constraints. A multitude of variables can play a role, and the complexity of forms across the tree of life may seemingly make the search for a taxon-specific theory of ageing a daunting task.

Here, theoretical models can provide guidance. Optimization approaches have been successfully applied to shed light on life history evolution [7-12]. Maynard Smith [11] emphasized in this context that ' $t$ the role of optimization theories in biology is not to demonstrate that organisms optimize. Rather, they are an attempt to understand the diversity of life'. It is in the spirit of Maynard Smith that the usefulness of such models can be appreciated. Evolution does not optimize [13], and, as pointed out by Gadgil and Bossert [7] in their seminal paper, 'the concept of an optimum or fittest genotype may be deceptive, since the definition of an optimum is as ephemeral as the environment on which it is based,... [but] it provides us with a powerful tool and is often the 
only useful model dealing with many biological problems'. Mathematical formulas cannot capture all complexities of biology, and biology is not physics with its exact laws. Yet, biology is not lawless. The overwhelming diversity of life history strategies follows universal principles that can potentially be captured by theoretical models.

To develop useful models, it is crucial to be aware of what limiting beliefs and assumptions explicitly or implicitly go into a model, because much of what theoretical models can provide depends on what initially is put in. Theory that became classic in gerontology has set powerful beliefs. For example, Hamilton [14] proved that the force of selection must inevitably decline over reproductive ages; thus, younger ages matter more to evolution than older ages. Therefore, as theories of the evolution of senescence $[1,14-16]$ assert, all species capable of repeated breeding must senesce, i.e. show patterns of increasing mortality and decreasing fertility from reproductive maturity onwards. The role of these theories has been so strong that empirical evidence of non-senescence $[2,4,5$, 17] has remained unaddressed. Theoretical models of life history theory explain the shape of reproductive patterns $[7-9,12]$, e.g. number and size of offspring, age at maturity or frequency of reproduction. Mortality in these models is assumed to be constant over adult ages [for example, see 18]. Models that explain constant and falling mortality have, until recently, been lacking. New approaches are needed that can explain patterns of senescence as well as non-senescence in both reproduction and mortality.

\section{Life History Models of Senescence}

The postulate of inevitable senescence [14] has constrained the scope of the models that have been developed. To aid future development of models that will be useful for building a taxon-specific theory of ageing, the discussion of several theoretical models below identifies elements in the assumptions that are crucial for capturing the shape of senescence patterns and in particular highlights those that distinguish senescence from non-senescence. Specifying life history trade-offs in this context is essential (box 2), because they are fundamental in driving the evolution of senescence.

\section{Box 2: Life History Trade-Offs}

Resources are limited. Plentiful reproduction and successful survival cannot always be attained simultaneously. Both processes require resources, so that higher survival generally comes at the cost of lower reproduction. Survival and reproduction can imply detrimental effects to themselves. For example, increasing reproduction at one age may cause damage (e.g. entailed by mating or producing progeny [19]) that reduces or prevents survival and/or reproduction at later ages. Resources that drive survival and reproduction need to be acquired at some costs, for instance the energy spent hunting prey or the increased risk of being attacked by a predator while collecting food in a risky environment. Resource acquisition trade-offs [20] in the field of optimal foraging theory [21-23], resource allocation trade-offs in life history theory [7-9, 12, 24] and both allocation and acquisition trade-offs together [25] have been extensively investigated by applying optimization models.

\section{Kirkwood and Holliday}

Assuming specific functional forms, Kirkwood and Holliday [26, see also 27] model mortality as increasing and fertility as decreasing exponential functions with age depending on a shared parameter, which captures the overall decay of the organism. The larger this parameter, the faster the decay in both survival and reproduction. Decay can be reduced by investment in repair. Damage thus affects the overall condition of the organism. The level of decay and thus maintenance is assumed to be constant over adult ages.

The trade-off between maintenance and reproduction is twofold; the higher the investment in maintenance, (1) the later reproduction starts and (2) the lower the initial, highest level of reproduction. Thus, the organism has to wait longer to get less profit per time unit, albeit over a longer period. The organism does not gain increased future reproductive potential by maintaining its physiological state but instead faces high costs in terms of late age of maturity and low levels of reproduction. With no prospects of increasing returns on investment in the future, this kind of trade-off favours the evolution of senescence.

The authors estimate the optimal level of maintenance by fitting the model to mouse data. The parameters that fit these data correspond to an optimal strategy of senescence. 


\section{Abrams and Ludwig}

In contrast to the previous model, Abrams and Ludwig [28] assume no particular functional forms for mortality or fertility when deriving the optimal list history choice. Instead, mortality is defined recursively. At every age, mortality increases by the amount of damage that accumulates. If at some age no damage occurs, mortality remains at its current level. Hence, age-specific mortality is assumed to be non-decreasing. The amount of damage accumulating at every age is assumed to be proportional to fertility, because more damage indicates that resources are diverted from survival and maintenance to reproduction. Initial fertility at age zero equals zero. As fertility increases, the amount of additional damage is assumed to decrease. Though the functional form of fertility is not specified, it is assumed to be fixed, i.e. it is not subject to evolution in this model. Notably, some baseline level of reproduction can occur without damage accumulating. This is an important feature of the trade-off because it allows parallel reproduction and maintenance of the organism.

The model is solved by deriving the conditions for a maximum in reproductive value (which is calculated as a specific function of any given fertility and mortality schedule) at every age with respect to the age-specific accumulation of damage. The endpoint condition depends on whether senescence or non-senescence evolves. For the case of increasing mortality, the endpoint condition simply corresponds to maximizing reproduction at the final age, which is defined as the age when mortality is so high that survival to the next age is negligible. Backward optimization finds the optimal strategy. In the case of non-senescence, mortality and fertility are maintained at a constant level. The dynamic programming equation can thus be solved since the reproductive value is constant.

Abrams and Ludwig find that sustenance (i.e. constant mortality and fertility over age) can be an optimal life history strategy. Given high age-specific survival, perfect repair is favoured if enough resources are available to ensure parallel reproduction and repair and if the gains in reproduction due to the marginal benefits of moving from a 'no-damage' to a 'damage' strategy are less than the costs of higher mortality. The authors note that this strategy may, however, not be stable if mechanisms other than the resource allocation strategy could affect mortality. For instance, the non-adaptive process of mutation accumulation $[1,14]$ could lower age-specific survival down to a point where the high-survival conditions would no longer be met and maintenance would become a suboptimal strategy. In the case of senescent strategies (i.e. life history strategies that, as a by-product, lead to increasing mortality and decreasing fertility over adult ages), numerical solutions are derived to determine what kind of patterns of fertility in this model imply what kind of optimal patterns of mortality, thereby exploring the nature of the trade-off between reproduction and repair.

The models of Abrams and Ludwig make a strong case for how alternative assumptions about the trade-off between reproduction and repair can lead to alternative patterns of mortality, including maintenance of the organism. Low risk of death and the option of efficient parallel maintenance and reproduction promote non-senescent strategies. Senescent strategies of various shapes are found when reproduction is costly in terms of damage accumulation.

The range of possible ageing patterns resulting from the Abrams-Ludwig models is wider than in the Kirkwood-Holliday model, because the functional forms of mortality and fertility in the condition for the optimal strategy are not specified. In particular, constant, i.e. non-ageing, patterns are not a priori excluded; the model allows for parallel maintenance (i.e. constant mortality) and non-zero reproduction. And indeed, the model finds sustenance a possible life history strategy. Note that the model assumes a direct trade-off between mortality and fertility mediated via a common 'damage' parameter.

\section{Kozlowski and Cichon}

Kozlowski and Cichon [29-32] have developed models that optimize resource allocation, assuming trade-offs between growth in size, repair of somatic damage and reproduction. As in the Abrams-Ludwig model, mortality is defined recursively as a non-decreasing function of age. At every age, damage accumulates depending on the amount of repair. Repair effects are modelled non-linearly. In contrast to the Abrams-Ludwig model, zero damage accumulation can only be achieved by investing all available energy in repair. Thus, zero damage comes at the cost of zero reproduction. The model assumptions exclude the option of parallel reproduction and maintenance; i.e., this specification of the trade-off implies that a non-senescent strategy cannot be evolutionarily optimal.

In an extended version of this model, Cichon and Kozlowski [31] assume that mortality not only depends on the accumulation of damage, but also on size. Note that in their model, the damage-dependent part of the mortality function is referred to as 'senescence' and the size-dependent part is interpreted as capturing 'extrinsic mortality', a distinction that touches on definitions and measure- 
ments in studies of senescence that are discussed elsewhere $[33,34]$. As size increases, mortality falls. Growth in size is costly; it competes for resources with repair and reproduction. The trade-off between growth and reproduction is modelled in a linear way, which yields optimal solutions of exclusive growth or reproduction. Therefore, this model captures species that stop growing when reproductive life starts. As in their initial models, full repair can only be achieved at the cost of zero reproduction. Optimal strategies therefore correspond to patterns of senescence.

The model is solved by backward programming, maximizing reproductive value at every age. The endpoint condition is assumed to be the age when the probability of surviving from birth to that age is less than one in a thousand. The maximum life span is an outcome of the model. Note that this specification of maximum life span implicitly assumes that remaining reproduction after this age, i.e. when less than one in a thousand survive, is negligible. Many trees and fish produce millions of seeds or eggs, and thousands of saplings and baby fish, but only few, much less than one in a thousand, survive to large sizes associated with high reproductive potential. Such strategies of parallel growth and reproduction are not favoured by the linear trade-off between reproduction and growth assumed in the model. For models that aim to describe species with indeterminate growth and increasing reproductive potential with age, such an endpoint condition would not be reasonable.

Note that for any optimal life history model of the type discussed in this article, it can be concluded that if there is an age after which future gains in reproduction are zero, then at this age it can never be optimal to invest in maintenance. Instead, it should always be optimal to invest everything that is left into reproduction. Thus, the existence of a final age of reproduction makes senescence an inevitable outcome of the model, at least senescence over ages close to this final age. Abrams and Ludwig get around this problem by distinguishing between two possible cases, i.e. maintenance versus senescence. In the maintenance case, the endpoint is simply given by the constant state of the organism reflected in constant birth and death rates and thus a constant reproductive value. In the senescence case, there is no problem assuming a finite age because if age-specific mortality increases, there clearly exists an age when survival to the next age becomes negligible and thus reproductive prospects are zero.

The models of Kozlowski and Cichon find that a variety of life history strategies can be optimal. These range from fast to slow senescence and from a short to long life span. All strategies, as implied by the trade-offs assumed, correspond to patterns of determinate growth and increasing mortality across adult life. Since mortality is assumed to be a non-decreasing function and constant mortality can only be achieved at the cost of zero reproduction, mortality must increase. Besides increasing mortality, another factor makes investment in future reproduction and survival sub-optimal; reproduction is constrained from increasing significantly in the future. Since reproduction is assumed to be proportional to an organism's size and since the linear trade-off between growth and reproduction implies that optimal strategies correspond to trajectories of exclusive growth and reproduction, i.e. growth ceases when reproduction begins, the size at reproductive maturity sets a limit to future reproductive potential.

\section{Mangel and Munch}

Initially developed to predict the evolution of compensatory growth [35], Munch and Mangel [36] present an optimization model to explain mortality patterns at prereproductive ages. Evolutionary theories of senescence predict that senescence should start at, but not before, reproductive maturity. In contrast to the models described above, here the trade-off between mortality and fertility is not direct but is instead mediated via physiological variables, i.e. size and damage. These variables describe the state of an organism at every age. Mortality is inversely related to size and proportional to the level of damage. The change in size and damage over time is regulated by the amount of foraging activity which is to be optimized. Mortality from predation increases with increasing foraging activity. The trade-offs in this model thus include energy acquisition costs.

More activity leads to higher energy intake, which increases the total amount of energy available for growth and the repair of damage, but more activity also increases the risk of predation and metabolic activity. Metabolism causes damage and requires energy. Growth increases size. A larger size lowers the risk of death and raises the amount of energy intake, but being big requires more energy for repair and higher metabolic activity. Most tradeoffs are non-linear; the functional forms assumed are motivated by the mass scaling of West et al. [37]. Parameters have mechanistic interpretations and most of them are theoretically measurable.

The model is solved backwards via a dynamic programming equation seeking the level of activity that maximizes reproductive value at every age. The endpoint 
is assumed to be the onset of reproduction. Residual reproductive value at maturity is assumed to be proportional to size and inversely related to damage. The model is analyzed across a wide range of parameters adopting a Monte Carlo approach.

Though not studying patterns over adult ages, i.e. ages when senescence is predicted to occur, this model provides insights into the evolution of ageing across juvenile ages. The model results show that mortality patterns across juvenile ages can be diverse, ranging from declining to broadly U-shaped trajectories. This diversity of results is enabled by the flexible nature of the assumed trade-offs. Trade-offs link reproduction and survival via physiological variables, are non-linear and do not a priori constrain mortality or fertility to be increasing or decreasing functions with age. The model of Mangel and Munch highlights the power of a state-based approach in modelling mortality and fertility.

\section{Vaupel, Baudisch and Colleagues}

Similar to the model of Mangel and Munch, these models $[2,5]$ assume that mortality, fertility and growth are determined by an organism's physiological state. A 'larger' state implies lower mortality and more resources available for maintenance, growth and reproduction, but also higher costs of maintenance. The change in state is driven by the balance between damage and repair. The physiological state can improve, deteriorate or remain the same over adult ages, depending on the allocation of resources between growth and reproduction. Damage occurs proportionally to the level of the state variable. Damage that occurs can be repaired. Thus, damage accumulation in this model is not a priori inevitable.

Alternative specifications of the state variable are assumed in several models. The simplest model is solved applying optimal control theory; the other models are solved using dynamic programming as done in the models discussed above. The optimal patterns of mortality and fertility result from the optimal resource allocation schedule over the course of life, which maximizes lifetime reproductive success. In contrast to the previous models, the endpoint condition is state specific. Energy available for the organism in the final state is equal to zero; mortality is therefore infinite and remaining reproduction is zero. The optimal strategy is found following a backward procedure from final to initial state. At every level of state the fraction of energy allocated to repair (as opposed to reproduction) is optimized.

The models find that age patterns of mortality and fertility can be diverse. They can go up or down or remain constant over adult ages. Thus, besides senescence, 'sustenance' and 'inverse senescence' - life histories of maintenance and improvement - can be optimal. Whether or not senescence is the optimal strategy crucially depends on the shape of the trade-off between reproduction and maintenance. In the 'vitality' model [2], concave tradeoffs lead to inverse senescence, linear trade-offs lead to sustenance and convex trade-offs lead to senescence. These results strongly motivate future investigation of the shape of the survival/reproduction trade-offs.

\section{Summary of Crucial Model Assumptions}

The discussion of the models presented above demonstrates how assumptions about the trade-off between survival and reproduction determine the range of possible senescence (or non-senescence) patterns. Important points in the model assumptions are (1) linearity versus non-linearity in the trade-offs, (2) inclusion or exclusion of mediating variables that determine mortality and fertility, (3) endpoint conditions of the optimization problem, (4) future returns to current investment reflected in the potential for indeterminate growth (which can also be interpreted more widely as the potential for acquisition of new skills, territory, access to mating partners, among others) and (5) constraints on the qualitative shape of mortality and fertility patterns, such as cumulative increase in mortality or exponential decline in fertility.

Table 1 summarizes those characteristics that seem crucial for whether non-senescence can be a potential model outcome. For the most part, these characteristics are not met by the models, likely due to the limiting beliefs of inevitable senescence. Building a 'damage ratchet' into the model does not allow previously accumulated damage to be removed, i.e. does not allow mortality to fall. Non-senescence can never result as an evolutionarily fittest strategy from a model if successful maintenance (constant mortality) in that setting can only be achieved at the cost of not being able to reproduce at all. And without an option for increasing reproductive potential, there is no incentive to invest in maintaining current structure, as there are no future benefits which may successfully compete with the benefits of reproducing right away.

Each of these models is useful in their own context. For the purpose of understanding the boundary between senescence and non-senescence, the characteristics listed in table 1 should be met. 
Table 1. Overview of models containing (+) or not containing $(-)$ assumptions that seem crucial to facilitate the evolution of non-senescence

\begin{tabular}{llll}
\hline & $\begin{array}{l}\text { Avoid the } \\
\text { damage ratchet }\end{array}$ & $\begin{array}{l}\text { Option of parallel } \\
\text { maintenance and } \\
\text { reproduction }\end{array}$ & $\begin{array}{l}\text { Option of increasing } \\
\text { reproductive potential } \\
\text { with adult age }\end{array}$ \\
\hline Kirkwood and Holliday [26, 27] & - & - & - \\
Abrams and Ludwig [28] & - & + & - \\
Kozlowski and Cichon [29-32] & - & - & NA \\
Mangel and Munch [35, 36] & + & + & + \\
Vaupel, Baudisch and colleagues [2,5] & + & & \\
\hline \multicolumn{2}{c}{ NA = Not applicable. } & &
\end{tabular}

\section{Discussion}

How realistic are these model assumptions? Which species can (1) escape the damage ratchet, (2) achieve maintenance and repair in parallel and (3) face increasing future reproductive potential?

Firstly, instead of repair, damage can efficiently be removed by a 'throw away, grow new' strategy. Modular body structures are indicative of such strategies $[2,3]$. Species capable of shedding their outer skeleton (e.g. arthropoda, nematoda) are good candidates to avoid the damage ratchet.

Secondly, to some extent, all species do at least some kind of maintenance in parallel to reproduction, but what is meant here is the ability to avoid an internal downward spiral of the physiological state. Such a spiral would be driven by a maintenance gap that emerges when physiological structure is grown that a priori could never be maintained even if the organism were to invest every bit of energy into repair and regeneration [38]. Trees, for example, have a clever way around this problem, at least to some extent. Though all the wood they build up eventually dies, it remains helpful to the whole organism without any need for maintenance or repair. Dead, inner layers of wood still provide the tree with stability, and bark at the outside shelters the layer of internal living tissue which continually keeps on growing. However, at some point trees may grow so tall that they reach a size where resource needs can no longer be met by resource acquisition $[39,40]$. Ways around the maintenance gap might have been found by species with flexible growth patterns, i.e. those capable of growth and shrinkage depending on resource availability [41]. Additionally, reproduction and growth need not always be contradictory aims. Vegetative reproduction might be a mode of growth that enables maintenance and reproduction at the same time, because newly grown structure can foster the survival of the individual but can also be separated off the individual body and become a contribution to reproduction [2].

Thirdly, increasing reproductive potential can result from continued growth over adult ages, learning and many other factors associated with, for example, more successful courtship, access to territory and resources. Many animal species show reproductive or survival improvement just after maturity [42], and some species are able to extend this phase throughout their lives, such as the netleaf oak Quercus rugosa or the tortoise Gopherus agassizii.

Understanding taxon-specific trade-offs is essential to shed light on taxon-specific ageing patterns. Challenges in identifying life history trade-offs are well known [12, $20,43]$. Predicted by theoretical considerations, it has recently been shown that the probability of detecting tradeoffs depends on the pace of life [44]. Theoretical models suggest that concavity versus convexity of trade-offs determines the boundary between senescence and non-senescence [2], which could guide empirical work. Likewise, empirical observations need to guide theoretical modelling. What variables matter? How should they be incorporated in a model?

Models not discussed here have been developed to study the evolution of ageing [45-48] that incorporate different variables and processes, including intergenerational transfers $[45,46]$ and density dependence [47]. Processes like intra- and inter-species resource transfers, density-dependent population regulation, sexual selection, competition, environmental variability and migration - the list goes on - affect ageing. Species differ with respect to numerous characteristics. We do not yet understand what variables are essential to include in models 
that explain the characteristic shape of ageing for different kinds of organisms: what explains the variation in ageing patterns across species? Does each species have its idiosyncrasies, or are there variables that generally explain certain shapes of trajectories?

Theory suggests the environmental hazard of death as one major candidate to mould the evolution of senescence in different populations. Specifically, the so-called Williams' hypothesis [16] predicts that senescence rates in a population should be higher, the riskier the environment. From a pace/shape point of view, this hypothesis is ambiguous, since senescence rates describe both aspects of ageing - change and direction as well as timing - in one variable. A higher environmental hazard will always reduce life span because as the average level of mortality goes up, life expectancy, which equals the inverse of average mortality (weighted by survival), necessarily goes down. A change in rates could simply reflect a faster pace of life; however, shape need not change $[6,49,50]$ (compare fig. 1). How ageing patterns across taxa are affected by the environment is a major question for future research that will rely on comparing data from wild and captive populations.

Empirical research on the evolution of senescence has recently experienced a strong surge of productivity [51, 52]. It is the right time to develop new models and theories that explain the emerging picture of ageing patterns across the tree of life, based on empirical evidence for how patterns of mortality and fertility are linked with underlying physiological changes in different taxa.

\section{References}

1 Medawar PB: An Unsolved Problem of Biol- 16 Williams GC: Pleiotropy, natural selection, ogy: Uniqueness of the Individual. London, H.K. Lewis, 1952, pp 44-70.

2 Baudisch A: Inevitable Senescence? Contributions to Evolutionary-Demographic Theory. Berlin, Springer, 2008.

3 Finch CE: Longevity, Senescence, and the Genome. Chicago, University of Chicago Press, 1990

4 Finch CE: Update on slow aging and negligible senescence - a mini-review. Gerontology 2009;55:307-313.

$\checkmark 5$ Vaupel JW, Baudisch A, Dolling M, Roach DA, Gampe J: The case for negative senescence. Theor Popul Biol 2004;65:339-351.

6 Baudisch A: The pace and shape of ageing. Methods Ecol Evol 2011;2:375-382.

7 Gadgil M, Bossert WH: Life historical consequences of natural selection. Am Nat 1970; 104:1-24.

$>8$ Pianka ER, Parker WS: Age-specific reproductive tactics. Am Nat 1975;109:453-464.

9 Roff DA: Life History Evolution. Sunderland, Sinauer Associates, 2002.

10 Schaffer WM: Selection for optimal life histories: the effects of age structure. Ecology 1974;55:291-303.

11 Smith JM: Optimization theory in evolution. Annu Rev Ecol Syst 1978;9:31-56.

12 Stearns SC: The Evolution of Life Histories. New York, Oxford University Press, 1992.

13 Parker GA, Smith JM: Optimality theory in evolutionary biology. Nature 1990;348:2733.

14 Hamilton WD: Moulding of senescence by natural selection. J Theor Biol 1966;12:1245.

15 Kirkwood TBL: Evolution of ageing. Nature 1977;270:301-304. and the evolution of senescence. Evolution 1957;11:398-411.

17 Martinez DE: Mortality patterns suggest lack of senescence in hydra. Exp Gerontol 1998;33:217-225.

18 Charnov EL: Reproductive effort is inversely proportional to average adult life span. Evol Ecol Res 2005;7:1221-1222.

19 Sgro CM, Partridge L: A delayed wave of death from reproduction in Drosophila. Science 1999;286:2521-2524.

20 Van Noordwijk AJ, De Jong G: Acquisition and allocation of resources: their influence on variation in life history tactics. Am Nat 1986;128:137-142.

21 Charnov EL: Optimal foraging, marginal value theorem. Theor Popul Biol 1976;9:129136.

-22 Pyke GH, Pulliam HR, Charnov EL: Optimal foraging - selective review of theory and tests. Q Rev Biol 1977;52:137-154.

23 Stephens DW, Krebs JR: Foraging Theory. Princeton, Princeton University Press, 1986.

24 Williams GC: Natural selection, the costs of reproduction, and a refinement of Lack's principle. Am Nat 1966;100:687-690.

25 De Jong G, Van Noordwijk AJ: Acquisition and allocation of resources: genetic covariances, selection and life histories. Am Nat 1992;139:749-770.

26 Kirkwood TBL, Holliday R: Aging as a consequence of natural selection; in Bittles $\mathrm{AH}$, Collins KJ (eds): The Biology of Human Ageing. Society for the Study of Human Biology, Symposium 25. Joint Symposium of the Society for the Study of Human Biology and the British Society for Research in Ageing. Cambridge, Cambridge University Press, 1986, pp 1-16.
27 Kirkwood TBL, Rose MR: Evolution of senescence: late survival sacrificed for reproduction. Philos Trans R Soc Lond B Biol Sci 1991;332:15-24.

28 Abrams PA, Ludwig D: Optimality theory, Gompertz' law, and the disposable soma theory of senescence. Evolution 1995;49:10551066.

29 Cichon M: Evolution of longevity through optimal resource allocation. Proc Biol Sci 1997;264:1383-1388.

30 Cichon M: Diversity of age-specific reproductive rates may result from ageing and optimal resource allocation. J Evol Biol 2001;14: 180-185.

31 Cichon M, Kozlowski J: Ageing and typical survivorship curves result from optimal resource allocation. Evol Ecol Res 2000;2:857870 .

32 Kozlowski J: Optimal allocation of resources explains interspecific life-history patterns in animals with indeterminate growth. Proc Biol Sci 1996;263:559-566.

33 Williams PD, Day T, Fletcher Q, Rowe L: The shaping of senescence in the wild. Trends Ecology Evol 2006;21:458-463.

34 Bronikowski AM, Promislow DEL: Testing evolutionary therories of aging in wild populations. Trends Ecol Evol 2005;20:271-273.

35 Mangel M, Munch SB: A life-history perspective on short- and long-term consequences of compensatory growth. Am Nat 2005;166:E155-E176.

36 Munch SB, Mangel M: Evaluation of mortality trajectories in evolutionary biodemography. Proc Natl Acad Sci USA 2006;103: 16604-16607.

37 West GB, Brown JH, Enquist BJ: A general model for ontogenetic growth. Nature 2001; 413:628-631. 
-38 Wensink MJ, van Heemst D, Rozing MP, Biogerontology WR: The maintenance gap: a new theoretical perspective on the evolution of aging. Biogerontology 2012;2:197-201.

- 39 Silvertown J, Franco M, Perez-Ishiwara R: Evolution of senescence in iteroparous perennial plants. Evol Ecol Res 2001;3:1-20.

40 Clark JR: Age-related changes in trees. J Arboric 1983;9:201-205.

41 Salguero-Gomez R, Casper BC: Keeping plant shrinkage in the demographic loop. J Ecol 2010;98:312-323.

42 Rebke M, Coulson T, Becker PH, Vaupel JW: Reproductive improvement and senescence in a long-lived bird. Proc Natl Acad Sci USA 2010;107:7841-7846.
43 Partridge L, Sibly R: Constraints in the evolution of life histories. Philos Trans R Soc Lond B Biol Sci 1991;332:3-13.

44 Hamel S, Gaillard JM, Yoccoz NG, Loison A, Bonenfant C, Descamps S: Fitness costs of reproduction depend on life speed: empirical evidence from mammalian populations. Ecol Lett 2010;13:915-935.

45 Chu CYC, Lee RD: The co-evolution of intergenerational transfers and longevity: an optimal life-history approach. Theor Popul Biol 2006;69:193-201.

46 Robson AJ, Kaplan HS: Why do we die? Am Econ Rev 2007;97:492-495.

47 Seymour RM, Doncaster CP: Density dependence triggers runaway selection of reduced senescence. PLoS Comput Biol 2007;3:e256.
48 Sozou PD, Seymour RM: To age or not to age. Proc Biol Sci 2004;271:457-463.

49 Caswell H: Extrinsic mortality and the evolution of senescence. Trends Ecol Evol 2007; 22:173-174.

50 Moorad JA, Promislow DEL: Evolution: aging up a tree? Curr Biol 2010;20:R406-R408.

51 Bouwhuis S, Choquet R, Sheldon BC, Verhulst S: The forms and fitness cost of senescence: age-specific recapture, survival, reproduction, and reproductive value in a wild bird population. Am Nat 2012;179:E15-E27.

52 Monaghan P, Charmantier A, Nussey D, Ricklefs R: Special feature: the evolutionary ecology of senescence. Funct Ecol 2008;22: 371-453. 